\title{
Bilateral rectus sheath block as the single anaesthetic technique for an open infraumbilical hernia repair
}

\author{
Kelvin How Yow Quek ${ }^{1}$, MBBS, MMed, Darren Shing Kuan $\underline{\text { Phua }}^{1}$, MBBS, MMed
}

\begin{abstract}
We present a case of an open surgical repair of an infraumbilical hernia, which was performed on a 45-year-old man categorised as American Society of Anesthesiologists class 4; he weighed $107 \mathrm{~kg}$, and had a body mass index of $34.2 \mathrm{~kg} / \mathrm{m}^{2}$ and nonischaemic cardiomyopathy (left ventricular ejection fraction of 20\%). Due to the patient's significant perioperative risks, the surgery was performed with the patient under ultrasonography-guided bilateral rectus sheath block; $15 \mathrm{~mL}$ of $1 \%$ lignocaine and $10 \mathrm{~mL}$ of $0.5 \%$ bupivacaine were deposited in the space between the rectus abdominis and posterior rectus sheath. The patient tolerated the surgery with minimal further sedation and additional analgesia. Rectus sheath block is a useful regional technique for periumbilical surgery, allowing surgery in high-risk patients while avoiding general anaesthesia and central neuraxial blockade. The use of real-time ultrasonographic guidance may reduce risks of peritoneal puncture, bleeding and visceral injury, while potentially increasing the rate of success.
\end{abstract}

Keywords: hernia, hernia repair, rectus sheath block, ultrasonography

\section{INTRODUCTION}

Rectus sheath block was first described by Schleich in 1899 as a means of facilitating surgery involving the anterior abdominal wall in adults. ${ }^{(1)}$ The central portion of the anterior abdominal wall is innervated by the ventral branches of the T7-T11 spinal nerve roots; these ventral branches lie between the rectus abdominis muscle and the posterior rectus sheath, and enter the rectus muscle near the midline. ${ }^{(2)}$ As the tendinous intersections of the rectus muscle are not fused to the posterior rectus sheath, local anaesthetic from a single injection site is able to spread cephalocaudally within this compartment. ${ }^{(3)}$

In recent times, rectus sheath block is more commonly used in paediatric patients. ${ }^{(4,5)}$ Its use in abdominal gynaecological procedures and umbilical hernia repair has also been described. ${ }^{(6-8)}$ In two previously reported cases, it was the sole anaesthetic used for elective umbilical surgery in high-risk patients with poor cardiovascular and physiological reserves. ${ }^{(9,10)}$ Few other cases have been documented in the literature.

We herein present a case of an open infraumbilical hernia repair, performed on an American Society of Anesthesiologists (ASA) class 4 patient under real-time ultrasonography-guided rectus sheath blocks, without general anaesthesia or central neuraxial blockade. This technique may be an invaluable alternative for anaesthesiologists managing high-risk patients requiring periumbilical surgery. Consent was obtained from the patient prior to the reporting of this case.

\section{CASE REPORT}

A 45-year-old man, weighing $107 \mathrm{~kg}$ and with a body mass index of $34.2 \mathrm{~kg} / \mathrm{m}^{2}$, presented for open repair of a symptomatic but nonobstructive infraumbilical hernia. The patient's concurrent comorbidities included type 2 diabetes mellitus and nonischaemic cardiomyopathy. The most recent transthoracic echocardiography revealed a moderately dilated left ventricle with an ejection fraction of $20 \%$, a severely dilated left atrium, moderate mitral regurgitation, and pulmonary hypertension with a pulmonary artery systolic pressure of $41 \mathrm{mmHg}$. Recent coronary angiography showed normal coronary arteries. The patient was hospitalised for congestive cardiac failure two months prior to the current presentation, and had been on anticoagulation therapy with warfarin secondary to atrial fibrillation. On surgical orders, warfarin was ceased five days prior to surgery. The patient was also on the cardiac transplant waiting list. He denied having any symptoms suggestive of obstructive sleep apnoea or gastro-oesophageal reflux disease. On examination, he was not found to be in overt cardiac failure, although there was mild bipedal pitting oedema. Open surgical repair for his symptomatic but nonobstructive infraumbilical hernia was expected to be uncomplicated.

The team decided to perform the surgery under bilateral rectus sheath block with judicious sedation in order to minimise haemodynamic instability. Central neuraxial blockade was not performed as there was the possibility of potentially significant haemodynamic swings and hypotension, which would have been detrimental to the patient. The patient was sedated with $1.5 \mathrm{mg}$ of intravenous midazolam prior to the procedure. Standard intraoperative monitoring, which included electrocardiography, pulse oximetry and noninvasive blood pressure monitoring, was performed. Oxygen supplementation via intranasal oxygen at $4 \mathrm{~L} / \mathrm{min}$ was commenced. Skin asepsis was ensured and the block was performed using an aseptic technique. The rectus sheath block was performed bilaterally

${ }^{1}$ Department of Anaesthesia, Changi General Hospital, Singapore

Correspondence: Dr Kelvin Quek, Consultant, Department of Anaesthesia, Changi General Hospital, 2 Simei Street 3, Singapore 529889. kelvin_quek@cgh.com.sg 

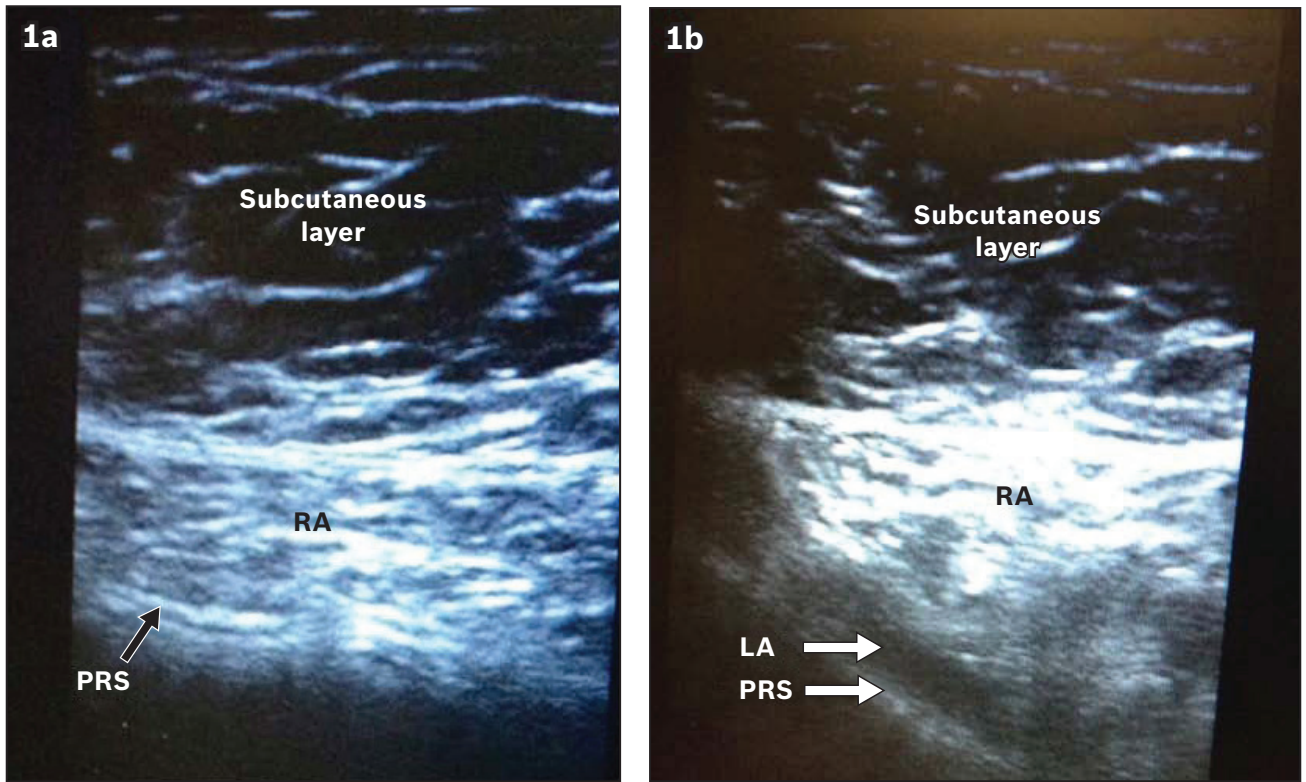

Fig. 1 US images show the rectus abdominis (RA) muscle and the posterior rectus sheath (PRS), (a) prior to, and (b) after local anaesthetic (LA) deposition.

with real-time ultrasonographic guidance (HFL38x/13-6 MHz Linear Array Transducer; Sonosite M-Turbo ${ }^{\mathrm{TM}}$, Bothell, WA, USA) using a 22G $(0.70 \mathrm{~mm} \times 50 \mathrm{~mm})$ Stimuplex ${ }^{\circledR} \mathrm{A}$ insulated needle (B. Braun, Melsungen, Germany) via an in-plane approach to the rectus sheath. $15 \mathrm{~mL}$ of $1 \%$ lignocaine, together with adrenaline $1: 200,000$, and $10 \mathrm{~mL}$ of $0.5 \%$ bupivacaine were deposited in the potential space between the rectus abdominis muscle and the posterior rectus sheath bilaterally (Figs. 1a \& b). A total of $30 \mathrm{~mL}$ of $1 \%$ lignocaine $(300 \mathrm{mg}$ ) and $20 \mathrm{~mL}$ of $0.5 \%$ bupivacaine (100 mg) were administered.

Surgery was commenced 20 mins after the block and low-dose intravenous propofol infusion at $50 \mathrm{mg} /$ hour was started for patient comfort and anxiolysis. At the start of the surgery, $50 \mu \mathrm{g}$ of intravenous fentanyl was administered to facilitate sedation while the surgeons supplemented the block by infiltrating the skin and subcutaneous tissues at the incision site with another $5 \mathrm{~mL}$ of $0.5 \%$ bupivacaine. The patient did not complain of pain at this juncture. A small transverse infraumbilical incision was made, and a $2 \mathrm{~cm} \times 2 \mathrm{~cm}$ defect containing the omentum was found. After dissection of the sac from the margin, fascial closure was performed, primarily with interrupted 1-0 prolene sutures, followed by subcutaneous and subcuticular closure.

The patient remained comfortable and haemodynamically stable throughout the surgery, with all vital signs remaining within $10 \%$ of baseline. No further drugs were required during the 45-min surgery. The patient was monitored in the postanaesthetic care unit. He did not require further analgesics and was discharged to the ward after an hour.

\section{DISCUSSION}

The anterior rami of the T7-T11 nerves provide sensory innervation to the rectus muscle and overlying skin. Local anaesthetic deposition within the posterior rectus sheath provides dense and predictable somatic anaesthesia to the anterior abdomen, sufficient for umbilical surgery or superficial surgery within the vicinity. Incisions in the suprapubic region may also derive some analgesic benefit, especially if supplemented with either ilioinguinal or transversus abdominis plane blocks. ${ }^{(11)}$ For surgeries deep to the peritoneum, however, there is usually deep visceral pain, for which systemic or epidural analgesia is required.

Compared to the transversus abdominis plane block, the rectus sheath block appears to provide denser analgesia of a shorter duration. Thus, the rectus sheath block is only useful for prolonged postoperative analgesia if continuous catheters are placed with regular dosing of local anaesthetics into the posterior rectus sheath. ${ }^{(12)}$

It has been suggested that continuous indwelling rectus sheath catheter placement possesses several advantages over epidural catheter placement, which is commonly performed for postoperative analgesia. ${ }^{(12)}$ Rectus sheath block is not associated with the physiological sympathectomy that accompanies central neuraxial blockade, hence avoiding haemodynamic fluctuations commonly seen with such a blockade. Additionally, unlike central neuraxial blockade, rectus sheath block is a viable option in the presence of relative coagulopathy, and recent use of antiplatelets or anticoagulants.

Continuous rectus sheath block also offers the major advantage of mobility. It combines excellent analgesia with preservation of limb strength and no mandatory connection to infusion devices, thus allowing patients to regain mobility early. This may translate into major clinical benefits such as the potential for a reduced incidence and severity of deep vein thrombosis and pulmonary embolism, reduced risk of atelectasis and respiratory infection, and minimal motor deconditioning. Moreover, the rectus sheath catheter can be 
safely inserted under general anaesthesia, avoiding patient discomfort and distress, which can occur during epidural insertion in an awake patient. This potentially increases patient acceptance of undergoing rectus sheath block. We did not consider a continuous rectus sheath catheter insertion in our patient as postoperative pain was not expected to be severe given the relatively small incision.

Traditionally, rectus sheath block is performed via a blind technique using the loss of resistance felt as the blunt (or block) needle is advanced through the fascial and muscle planes. This technique has, however, remained underused, primarily due to concerns about the accuracy of needle placement, particularly in relation to the underlying peritoneal structures.

Ultrasonography-guided rectus sheath blocks offer significant advantages, such as providing noninvasive imaging of the anatomy, facilitating real-time needle guidance, and allowing observation of the local anaesthetic spread within the correct tissue plane. ${ }^{(13)} \mathrm{A}$ recent systematic review of ultrasonography-guided truncal blocks strongly recommended the use of ultrasonographic guidance for rectus sheath block in order to increase its success rate. ${ }^{(3)}$ Even though studies to date lack the statistical power to demonstrate any safety advantage conferred with the use of ultrasonography, the visualisation of real-time sonoanatomy, especially with in-plane techniques, are likely to reduce the incidence of inadvertent needle entry into the peritoneum, blood vessel or bowel, and increase the rate of successful nerve blocks.

With ultrasonography becoming increasingly available in anaesthetic practice, we believe this often overlooked technique is an important component of every anaesthesiologist's skill set. Rectus sheath block is likely to be useful in selected patients requiring simple periumbilical surgery, primarily those in whom the risks accompanying general anaesthesia or central neuraxial blockade are high, as was encountered in our case.

\section{REFERENCES}

1. Schleich DL. Schmerzlose Operationen. 4th ed. Berlin: Springer Verlag, 1899: 240-58.

2. Snell R. Clinical anatomy. 8th ed. Baltimore: Lippincott Williams \& Wilkins, 2008.

3. Abrahams MS, Horn JL, Noles LM, Aziz MF. Evidence-based medicine: ultrasound guidance for truncal blocks. Reg Anaesth Pain Med 2010; 35:S36-42.

4. Ferguson S, Thomas V, Lewis I. The rectus sheath block in paediatric anaesthesia: new indications for an old technique? Paediatr Anaesth 1996; 6:463-6.

5. Willschke $\mathrm{H}$, Bösenberg A, Marhofer $\mathrm{P}$, et al. Ultrasonography-guided rectus sheath block in paediatric anaesthesia--a new approach to an old technique. Br J Anaesth 2006; 97:244-9.

6. Azemati S, Khosravi MB. An assessment of the value of rectus sheath block for postlaparoscopic pain in gynecologic surgery. J Minim Invasive Gynecol 2005; 12:12-5.

7. Crosbie EJ, Massiah NS, Achiampong JY, Dolling S, Slade RJ. The surgical rectus sheath block for post-operative analgesia: a modern approach to an established technique. Eur J Obstet Gynecol Reprod Biol 2012; 160:196-200.

8. Gurnaney HG, Maxwell LG, Kraemer FW, et al. Prospective randomized observer-blinded study comparing the analgesic efficacy of ultrasoundguided rectus sheath block and local anaesthetic infiltration for umbilical hernia repair. Br J Anaesth 2011; 107:790-5.

9. Muir J, Ferguson S. The rectus sheath block - well worth remembering. Anaesthesia 1996; 51:893-4.

10. Phua DS, Phoo JW, Koay CK. The ultrasound-guided rectus sheath block as an anaesthetic in adult paraumbilical hernia repair. Anaesth Intensive Care 2009; 37:499-500.

11. Yentis SM, Hills-Wright $P$, Potparic O. Development and evaluation of combined rectus sheath and ilioinguinal blocks for abdominal gynaecological surgery. Anaesthesia 1999; 54:475-9.

12. Webster K. Ultrasound guided rectus sheath block - analgesia for abdominal surgery. Update in Anaesthesia 2010; 26:12-17.

13. Awad IT, Chan V. Ultrasound imaging of peripheral nerves: a need for a new trend. Reg Anaesth Pain Med 2005; 30:321-3. 